\title{
Autoantibodies against EPCR are found in antiphospholipid syndrome and are a risk factor for fetal death
}

Verónica Hurtado, Ramón Montes, Jean-Christophe Gris, María L. Bertolaccini, Álvaro Alonso, Miguel A. Martínez-González, Munther A. Khamashta, Kenji Fukudome, David A. Lane, and José Hermida

\begin{abstract}
The antiphospholipid syndrome (APS) is associated with thrombosis and fetal death but the pathologic mechanisms are poorly understood. Since endothelial protein $\mathrm{C}$ receptor (EPCR) plays a role in the anticoagulant system and in placental development, we hypothesized that anti-EPCR autoantibodies may be involved in clinical manifestations of APS and in fetal loss. The levels of immunoglobulin $M(\lg M)$ and $\lg G$ anti-EPCR autoantibodies were analyzed by enzymelinked immunosorbent assay (ELISA) in 43
\end{abstract}

patients with APS and 43 controls. AntiEPCR levels were higher in APS patients than in controls. Interestingly, one of the IgM anti-EPCR autoantibodies inhibited the generation of activated protein $\mathbf{C}$ on endothelium. Since markedly high anti-EPCR levels were found in women with fetal death, 87 patients with a first episode of unexplained fetal death were subsequently analyzed and their anti-EPCR levels were compared with 87 matched controls. We found that antiEPCR autoantibodies constitute an indepen- dent risk factor for a first fetal death episode: the adjusted odds ratios (ORs) for anti-EPCR autoantibodies above the 95th percentile were $\mathbf{2 3 . 0}$ (95\% confidence interval $[\mathrm{Cl}$, 2.0-266.3) for IgM and $6.8(95 \% \mathrm{Cl}$, 1.2-38.4) for IgG. Anti-EPCR autoantibodies can be detected in APS patients and are independent risk factors for fetal death. (Blood. 2004;104:1369-1374)

() 2004 by The American Society of Hematology

\section{Introduction}

Antiphospholipid syndrome (APS) is characterized by vascular thrombosis and complications of pregnancy (fetal death, premature birth, or multiple spontaneous abortions) associated with the presence of antiphospholipid antibodies (APLs). ${ }^{1}$ These antibodies are heterogeneous and recognize a variety of combinations of phospholipids, phospholipid-binding proteins, or both. ${ }^{2}$ The most commonly detected subgroups of antiphospholipid antibodies are lupus anticoagulant antibodies (LACs) and anticardiolipin antibodies (aCLs). Other antiphospholipid antibodies are directed against phospholipids other than cardiolipin or against phospholipidbinding proteins like $\beta 2$-glycoprotein I or annexin V. ${ }^{3,4}$ However, the mechanisms linking the presence of APLs with thrombosis and pregnancy complications such as fetal death are poorly understood.

Activated protein $\mathrm{C}$ (APC) is a major regulatory protein of the coagulation cascade. Protein C (PC), the zymogen of APC, is activated by thrombin bound to thrombomodulin on cell surfaces. APC, working in concert with its nonenzymatic cofactor protein S, exerts its anticoagulant function by proteolyzing activated factors $\mathrm{V}$ and VIII. ${ }^{5}$ Genetic and acquired defects of thrombomodulin, PC, and protein $\mathrm{S}$ have been identified in patients with venous and/or arterial thrombosis, and antibodies against these molecules have been related with APS. ${ }^{6}$ Endothelial PC/APC receptor (EPCR) is an endothelial cell membrane glycoprotein that is tightly bound to a phospholipid and binds PC and APC. ${ }^{7,8}$ EPCR is mainly expressed in large vessels, especially arteries, and in the syncytiotrophoblast as well. ${ }^{9,10} \mathrm{PC}$ binding to EPCR notably enhances its activation by the thrombin-thrombomodulin complex. ${ }^{11}$ APC binding to EPCR allows APC-dependent tethering of protease-activated receptor-1, which blocks the apoptosis of endothelial and probably placental cells. ${ }^{10,12-14}$ In addition, growing evidence supports a role for EPCR in pregnancy maintenance, since EPCR knockout mice experience placental thrombosis and early embryonic mortality. ${ }^{15}$ This fact, together with the nature of EPCR as a phospholipid-binding protein, its function as anticoagulant and cell protector, its involvement in the development of placenta, and its localization on the surface of vessels and placenta, makes this molecule a possible target for APL associated with fetal death and thrombotic disease.

We investigated the presence of anti-EPCR autoantibodies in patients with APS. The results obtained made us suspect that a relationship between these antibodies and fetal death may exist. For this reason, we subsequently studied the association of anti-EPCR autoantibodies with first fetal death in a matched case-control study. We also studied the effect of these autoantibodies on APC generation on the endothelial surface. Our results support that anti-EPCR autoantibodies constitute a risk factor for a first fetal death episode. Impaired PC activation on cell surfaces could be one of the ways by which these antibodies exert their pathologic effects.
From the Haematology Department and the Division of Cardiovascular Pathophysiology, Laboratory of Thrombosis and Haemostasis, Clínica Universitaria/School of Medicine, Applied Medical Research Centre, University of Navarra, Pamplona, Spain; Haematology Laboratory, University Hospital, Nîmes, France; Lupus Research Unit, The Rayne Institute, St Thomas Hospital, London, United Kingdom; Department of Epidemiology and Public Health, School of Medicine, University of Navarra, Pamplona, Spain; Department of Immunology, Saga Medical School, Saga, Japan; and Department of Haematology-Division of Investigative Science, Hammersmith Campus, Imperial College, London, United Kingdom.

Submitted March 2, 2004; accepted April 29, 2004. Prepublished online as Blood First Edition Paper, May 18, 2004; DOI 10.1182/blood-2004-03-0793.
Supported through the Unión Temporal de Empresas (UTE) project Centro de Investigación Médica Aplicada (CIMA). Also supported by Servicio Navarro de Salud grants 18/2002 and 3/2004; Instituto de Salud Carlos III, Ministerio de Sanidad y Consumo, Spain, grant 01/0247; and the British Heart Foundation.

Reprints: José Hermida, Haematology Department, Clínica Universitaria, University of Navarra, Avenida Pío XII, 36, 31008 Pamplona, Spain; e-mail: jhermida@unav.es.

The publication costs of this article were defrayed in part by page charge payment. Therefore, and solely to indicate this fact, this article is hereby marked "advertisement" in accordance with 18 U.S.C. section 1734.

(c) 2004 by The American Society of Hematology 


\section{Patients, materials, and methods}

\section{APS patients and controls}

Cases were 43 consecutive patients (ages 17-67 years; median age, 43 years; 39 women and 4 men) diagnosed with APS according to international criteria $^{16,17}$ between February 1998 and March 2002 at the St Thomas' Hospital (London, United Kingdom). All of them were identified by having LACs and a personal history of venous thrombosis $(n=17)$, arterial thrombosis $(n=13)$, or both $(n=13)$. Serum samples were drawn at least 3 months after the last thrombotic episode and stored at $-80^{\circ} \mathrm{C}$ until tested for anti-EPCR autoantibodies. The control group was composed of 43 healthy volunteers with no history of thrombosis or LACs. All patients and controls had given their informed consent for participation in the study. The study was approved by the Ethics Committees or Sir Thomas' Hospital and Clínica Universitaria of the University of Navarra.

\section{Women with fetal death and controls}

A matched case-control study on fetal death was carried out at the University Hospital of Nîmes (France) and in clinics belonging to the same obstetric network between September 1996 and September 2002. This study was part of the Mediterranean Abnormal Pregnancy Study Program that led to the previously published Nîmes Obstetricians and Haematologist Study (NOHA). ${ }^{18}$

We included 87 consecutive women, 19 to 31 years old (median age, 27 years), with a first episode of fetal death from the 10th week of amenorrhea that had occurred during their last pregnancy. Reasons for exclusion included the following: any thrombotic antecedents, any chromosomal abnormality in the conceptus karyotype, any morphologic malformation in the fetus, chronic infectious disease or any known systemic disease, any antecedents of other type of pregnancy with poor outcome (early embryo loss before the 10th week of gestation, eclampsia, intrauterine fetal growth restriction), diabetes mellitus, or lack of adherence to study procedures. The fetal death had occurred during the first pregnancy in 58 women, during the second pregnancy in 21 women, and during the third pregnancy in the remaining 8 women; the fetal death had occurred between the 10th and the 22 nd weeks in 75 women and between the 22nd and the 36th weeks in the remaining 12 women.

A control group of 87 healthy mothers, matched for age, number of pregnancies, and time elapsed since the end of the last pregnancy (all negative for thrombotic history and chronic infectious diseases), was simultaneously recruited during the same period of time as the patients, from consecutive women attending the outpatient Department of Gynaecology at the same hospital for a general medical examination.

The study was approved by the Ethics Committee of the University Hospital of Nîmes and the Clínica Universitaria of the University of Navarra, and informed consent was obtained from all participants. Inclusion of cases and controls and blood collection took place at least 6 months after fetal death. Blood samples were collected, processed, and stored at $-80^{\circ} \mathrm{C}$ according to standard procedures.

\section{Expression of soluble EPCR}

We amplified the soluble EPCR (sEPCR) sequence, comprising the extracellular domain without its signal peptide and transmembrane and intracellular domains (residues 1-193, mature protein numbering), ${ }^{19}$ by polymerase chain reaction with primers that added ClaI and NotI restriction sites at the $5^{\prime}$ and $3^{\prime}$ ends, respectively: agcttggcatatcgattagccaagacgcctcagatg and tattctatgcggccgccgaagtgtaggagcggcttg. These modifications allowed us to ligate the sEPCR sequence into the Cla and Not sites of the plasmid pPICZ $\alpha \mathrm{C}$ (Invitrogen, Paisley, United Kingdom) following the Saccharomyces cerevisiae $\alpha$-factor secretion signal that allows for efficient secretion of many proteins from Pichia pastoris. The insert was cloned in-frame with a myc epitope followed by 6 histidines (His) present in the vector in order to express the sEPCR fused with myc and 6 His at the C-terminal end. Yeast was transformed with the linearized vector, thus integrating the sEPCR coding sequence into the endogenous methanol- responsive promoter by homologous recombination. As the pPICZ $\alpha \mathrm{C}$ vector encodes for the zeocin resistance gene, zeocin-resistant colonies of Pichia pastoris were screened for sEPCR expression induced by $1 \%$ methanol. The most efficient sEPCR-expressing colony was selected for large-scale production.

\section{Purification of recombinant sEPCR}

Recombinant sEPCR was purified from culture supernatants using a 3-step purification. The sample was loaded onto a 5-mL HiTrap chelating high-performance (HP) column (Amersham Biosciences, Little Chalfont, United Kingdom) loaded with copper. The bound fraction was eluted with $50 \mathrm{mM}$ EDTA (ethylenediaminetetraacetic acid), dialyzed against $20 \mathrm{mM}$ Tris- $\mathrm{HCl}(\mathrm{pH} 7.5)$, and then subjected to anion exchange using a Resource Q column (Amersham Biosciences) and a salt gradient from 0 to $300 \mathrm{mM}$ $\mathrm{NaCl}$ over 20-column volumes. The sEPCR-containing eluted fractions were applied to a Superdex 75-HR10/30 column (Amersham Biosciences). The concentration of purified protein was determined using the bicinchoninic acid (BCA) total protein assay (Pierce, Rockford, IL).

\section{Enzyme-linked immunosorbent assay (ELISA) for the determination of anti-EPCR autoantibodies}

Ninety-six-well microplates (Costar, Acton, MA) were coated with $0.15 \mu \mathrm{g}$ anti-myc monoclonal antibody (MoAb; Invitrogen) to anchor sEPCR, preserving its extracellular epitopes (direct sEPCR binding to wells occludes several epitopes). ${ }^{11}$ After washing with Tris-buffered saline (TBS; $20 \mathrm{mM}$ Tris, $150 \mathrm{mM} \mathrm{NaCl}, 0.05 \%$ Tween-20 [pH 7.4]), nonspecific binding sites were blocked with $3 \%$ (wt/vol) bovine serum albumin (BSA) in TBS. One hundred microliters per well of $1 \mu \mathrm{g} / \mathrm{mL}$ recombinant sEPCR in TBS supplemented with $1 \%$ BSA (TBS-BSA) were incubated at room temperature (RT) for 4 hours. Subsequently, $100 \mu \mathrm{L}$ of a 1:100 dilution of the sample (plasma or serum) in TBS-BSA was incubated overnight at $4^{\circ} \mathrm{C}$. Parallel blank wells were included with TBS-BSA without plasma or serum. Bound immunoglobulin $\mathrm{M}(\mathrm{IgM})$ and $\mathrm{IgG}$ anti-EPCR autoantibodies were detected with either a peroxidase-conjugated murine polyclonal antihuman IgM (Zymed, South San Francisco, CA) or an alkaline phosphataseconjugated murine polyclonal antihuman IgG (Zymed). After 2 hours at RT and extensive washing, the plates were developed with either $100 \mu \mathrm{L}$ of $0.4 \mathrm{mg} / \mathrm{mL}$ o-phenylenediamine (IgM; Kodak, Rochester, NY) or 100 $\mu \mathrm{L}$ of $1 \mathrm{mg} / \mathrm{mL}$ 4-nitrophenil phosphate (IgG; Sigma, St Louis, MO) and read at 492 or $405 \mathrm{~nm}$, respectively, in an iEMS Reader (Labsystems, Helsinki, Finland).

To allow comparisons among plates, we chose a sample to be assayed in every plate (standard sample), which thus allowed us to introduce a correction factor. Arbitrary units (AUs) were defined as follows: for each patient sample (problem sample), the specific absorbance was calculated by subtracting the absorbance of blank wells and then multiplying by 1000 and by a correction factor corresponding to the ratio between the absorbance of the standard sample assayed in a given plate (reference plate) and in the plate where the problem sample is assayed. Samples from patients were assayed together with those from their matched controls in the same plate to minimize differences due to the interassay variability. All samples were assayed twice. The intra-assay and interassay coefficients of variation were evaluated with 5 samples tested 5 and 3 times, respectively, and were always found to be lower than $5 \%$ and $10 \%$, respectively.

\section{Purification of anti-EPCR autoantibodies}

IgM fraction from serum or plasma samples containing high titers of $\operatorname{IgM}$ anti-EPCR was isolated using a HiTrap $N$-hydroxy-succinimide (NHS)activated HP column (Amersham Biosciences) where a murine antihuman $\mathrm{IgM} \mathrm{MoAb}$ had previously been immobilized. ${ }^{20} \mathrm{IgG}$ fraction from serum or plasma samples containing IgG anti-EPCR was isolated using a HiTrap protein G HP column (Amersham Biosciences) according to the manufacturer's instructions.

\section{APC generation on cultured endothelial cells}

Fifty thousand EA.hy926 cells ${ }^{11}$ (endothelium-derived cell line expressing thrombomodulin and EPCR, kindly supplied by Dr C. J. Edgell, University 
of North Carolina) were incubated with $0.02 \mathrm{U} / \mathrm{mL}(0.17 \mathrm{nM})$ thrombin (ERL, Swansea, United Kingdom) and increasing concentrations of PC (kindly supplied by Baxter, Deerfield, IL) ranging between 50 and $1000 \mathrm{nM}$ in a 20-mM Tris buffer ( $\mathrm{pH}$ 7.4) supplemented with $150 \mathrm{mM} \mathrm{NaCl}, 5 \mathrm{mM}$ $\mathrm{CaCl}_{2}, 0.6 \mathrm{mM} \mathrm{MgCl}_{2}, 1 \% \mathrm{BSA}, 0.01 \%$ Tween-20, and $0.02 \% \mathrm{NaN}_{3}$. After 45 minutes, lepirudin (Schering AG, Berlin, Germany) was added at 0.2 $\mu \mathrm{M}$ to inhibit thrombin, and 4 minutes afterward the chromogenic substrate S-2366 (Chromogenix, Milan, Italy) was added at $0.4 \mathrm{mM}$ and its proteolysis by APC was kinetically monitored at $405 \mathrm{~nm}$ in the iEMS Reader. The data curve fitting the Michaelis-Menten equation was performed using Enzfitter software (Biosoft, Cambridge, United Kingdom), which calculated the $K_{\mathrm{m}}$ and $V_{\max }$ values of PC activation under these conditions. Where required, $20 \mu \mathrm{g} / \mathrm{mL}$ RCR-252 MoAb, which blocks PC-EPCR binding, ${ }^{21}$ or anti-EPCR autoantibodies from patients were added.

\section{Preparation of a sEPCR affinity column}

Two milligrams of recombinant sEPCR was coupled to a HiTrap NHSactivated HP column (Amersham Biosciences) following the manufacturer's instructions. Since the sEPCR bound to the column retained its ability to bind PC, it probably retained the native conformation including the epitopes recognized by the autoantibodies. Thus, this column is suitable for anti-EPCR depleting.

\section{Statistical methods}

In the APS case-control study, the comparison between cases and controls for frequency of high levels of IgM and IgG anti-EPCR was performed with a chi-square test.

In the fetal death matched case-control study, comparison between cases and controls for continuous and categoric variables was performed with a $t$ test for paired samples and McNemar test, respectively. Association between levels of IgM and IgG anti-EPCR with LACs and IgM aCLs was assessed with correlation coefficients for continuous variables and MannWhitney $U$ test for categoric variables.

In order to evaluate the risk of fetal death associated with high levels of IgM and IgG anti-EPCR, a conditional logistic regression analysis was used with matched pairs of cases and controls. The main independent variables were levels of $\operatorname{IgM}$ and $\operatorname{IgG}$ anti-EPCR categorized according to the distribution of these antibodies in controls. We used different cutoff points to determine the levels associated with a higher risk. Univariate and multivariate analysis were performed, adjusting for known risk factors of fetal death. The levels of anti-EPCR autoantibodies were also divided into quartiles to assess a relationship between this variable and risk of fetal death (dose response). Tests for trend in fetal death across quartiles of anti-EPCR were assessed in conditional logistic models using a continuous variable with the median values for each quartile of the antibodies. Interaction (effect modification) was assessed introducing product terms in the conditional logistic models. The Mann-Whitney $U$ test was used to compare APC generation in the presence and absence of anti-EPCR autoantibodies.

\section{Results}

More than $5 \mathrm{mg}$ of sEPCR could be purified from a Pichia culture. By sodium dodecyl sulfate-polyacrylamide gel electrophoresis (SDS-PAGE) and Western blot analysis, sEPCR appeared as a single band. The PC activation by thrombin on the EA.hy926 cells $\left(K_{\mathrm{m}}=69 \pm 18 \mathrm{nM}\right)$ was hampered in the presence of $2 \mu \mathrm{M}$ sEPCR (apparent $\mathrm{K}_{\mathrm{i}}=75 \pm 4 \mathrm{nM}$ ), suggesting that our recombinant sEPCR binds to PC with similar efficiency to native EPCR. ${ }^{22,23}$ Furthermore, the sEPCR was able to inhibit the anticoagulant activity of APC in a modified clotting assay, as expected ${ }^{22}$ (data not shown). This evidence strongly suggests that our recombinant sEPCR is folded in the correct conformation, allowing us to use it to detect autoantibodies against EPCR.

\section{Anti-EPCR autoantibodies in APS patients}

The median values of both IgM and IgG anti-EPCR were higher among APS cases than among controls (57 AUs vs 45 AUs and 75 AUs vs 72 AUs, respectively; Figure 1). Even if the difference between mean levels was not statistically significant, $21 \%$ of APS patients had either IgM or IgG anti-EPCR levels exceeding the 97th percentile compared with 5\% in control subjects $(P=.024)$. Three patients displayed very high levels of IgM anti-EPCR (patient A $=407$ AUs, patient B = 301 AUs, patient $\mathrm{C}=293 \mathrm{AUs}$ ), and 2 patients had high IgG anti-EPCR levels (patient $\mathrm{D}=230$ AUs, patient $\mathrm{E}=220$ AUs). All 5 of these patients were women with a previous history of thrombosis. Two women carrying IgM anti-EPCR (patients A and B) had suffered from multiple episodes of fetal death.

\section{Biochemical characterization of anti-EPCR autoantibodies}

IgM fractions from patients $\mathrm{A}, \mathrm{B}$, and $\mathrm{C}$, and $\mathrm{IgG}$ fractions from patients $\mathrm{D}$ and $\mathrm{E}$ were purified from serum. IgM fraction of patient $\mathrm{C}$ remarkably inhibited the thrombin-induced APC generation by EA.hy926 cells in a dose-dependent manner (maximum effect about $80 \%$ reduction of PC activation; $P=.02$ ), an effect comparable to that obtained with RCR252 (Figure 2). The decrease in the APC generation was due to an increase in the $K_{\mathrm{m}}$ rather than $V_{\max }$, compatible with ablation of the EPCR-enhancing effect on activation (Figure 3). To demonstrate that this effect was due to a specific IgM against EPCR, the patient $\mathrm{C}$ sample was anti-EPCR autoantibody-depleted by loading it onto the sEPCR affinity column. The sample lost the inhibitory capacity on APC generation, indicating that a specific anti-EPCR autoantibody was responsible for this phenomenon (Figure 2).

\section{Anti-EPCR autoantibodies in women with fetal death}

Since a relationship between anti-EPCR autoantibodies and fetal death could be hypothesized from the results presented in the APS study, we focused our analysis on the association between antiEPCR and fetal death in a group of 87 women with a first episode of unexplained fetal death without previous history of venous and/or arterial thrombosis and 87 healthy age-matched controls. Table 1 shows the frequencies of risk factors classically related with fetal death together with the frequencies of $\operatorname{IgM}$ and $\mathrm{IgG}$ anti-EPCR found in patients and controls. The 95th percentile of the IgM anti-EPCR in the control group was 99 AUs. Eighteen percent of patients had values exceeding this cutoff compared with only $3 \%$ of controls. The crude relative risk, estimated by the matched odds ratio (OR), for fetal death in patients with $\operatorname{IgM}$ anti-EPCR levels above the 95th percentile was $14.0(95 \%$

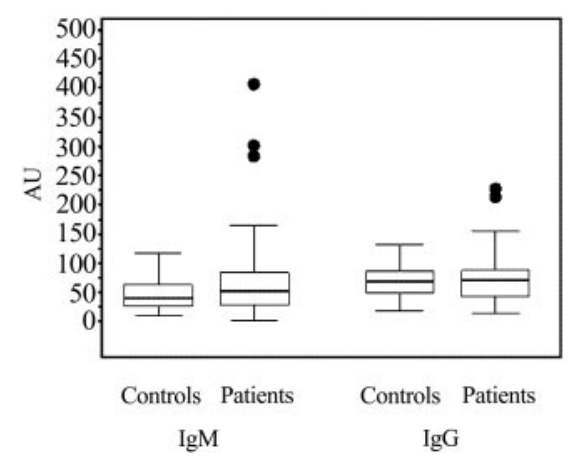

Figure 1. Anti-EPCR autoantibodies in APS patients and controls. Anti-EPCR levels are shown (median, interquartile range, range, and outliers). IgM anti-EPCR: controls, median $=45$ AUs; patients, median $=57$ AUs. IgG antibodies: controls, median $=72$ AUs; patients, median $=75$ AUs. 


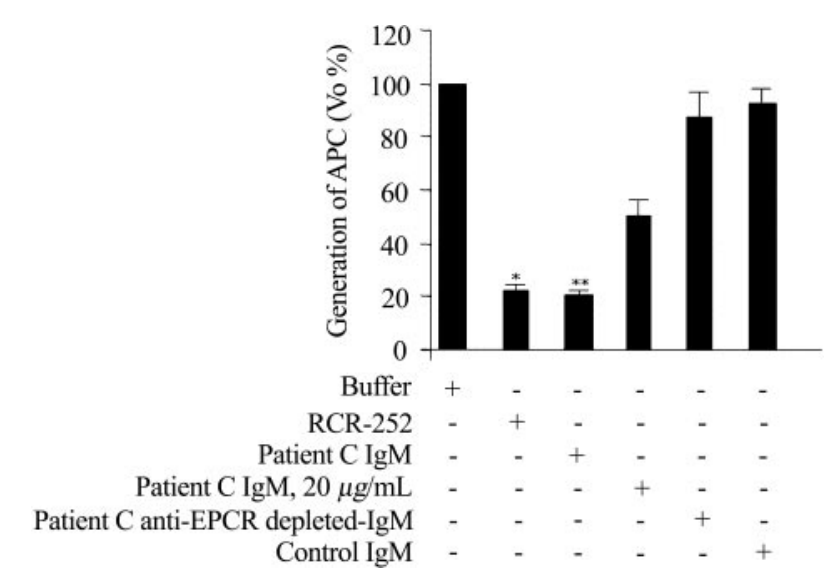

Figure 2. Effect of anti-EPCR antibodies on APC generation by endothelial cells. Confluent EA.hy926 cells were incubated with buffer or the indicated $A b$ (RCR-252 at $20 \mu \mathrm{g} / \mathrm{mL}$, the different patients' fractions at $45 \mu \mathrm{g} / \mathrm{mL}$ except indicated) and the initial rate (Vo) of thrombin-dependent activation of $50 \mathrm{nM} \mathrm{PC}$ was determined. For each condition, 2 to 4 independent experiments were performed. The mean \pm standard deviation of $\%$ Vo with respect to the Vo obtained in the absence of antibodies is represented. ${ }^{*} P=.013,{ }^{* *} P=.02$, with respect to the buffer condition.

confidence interval [95\% CI], 1.8-106.4) compared with those who had a lower value. When the cutoff was set at the 90th percentile (83 AUs), the OR was 5.2 (95\% CI, 1.8-15.3).

The 95th percentile of the IgG anti-EPCR levels in the control group was 94 AUs. Fifteen percent of patients had values exceeding this cutoff compared with $5 \%$ of controls. The crude OR for fetal death in patients with $\operatorname{IgG}$ anti-EPCR levels above the 95th percentile was 4.3 (95\% CI, 1.2-15.2). When the cutoff was set at the 90th percentile (80 AUs), the OR was 2.3 (95\% CI, 0.9-5.6).

In order to improve accuracy in the analysis of anti-EPCR autoantibodies as risk factors for fetal death, we performed a separate analysis adjusting for putative confounding variables (Table 2). Due to the sample size, the data were sparse and we were not able to adjust simultaneously for factor V Leiden (FVL) and LACs in the same model. For this reason we considered 2 different models: model 1, in which the adjustment was performed for APLs (ie, LACs and aCLs), prothrombin G20210A, and, in the case of

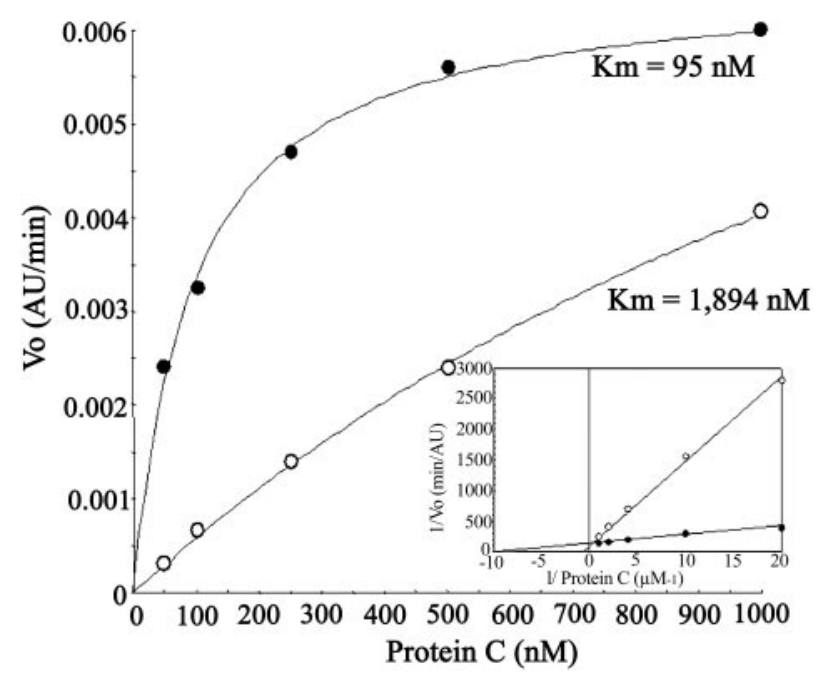

Figure 3. Effect of patient C anti-EPCR autoantibody on kinetic parameters of APC generation by endothelial cells. Thrombin-catalyzed protein $C$ activation on EA.hy926 cells measured in the absence $(\bullet)$ or presence $(\bigcirc)$ of patient $C$ IgM fraction at $45 \mu \mathrm{g} / \mathrm{mL}$. The $K_{\mathrm{m}}$ for each condition is indicated. The Lineweaver-Burk plot in the inset shows that the $V_{\max }$ in both conditions is essentially equal.
Table 1. Univariate OR and their $95 \% \mathrm{Cl}$ for fetal death associated with different variables studied

\begin{tabular}{lccccc}
\hline & $\begin{array}{c}\text { Fetal death; } \\
\mathbf{n}=\mathbf{8 7}\end{array}$ & $\begin{array}{c}\text { Controls; } \\
\mathbf{n}=\mathbf{8 7}\end{array}$ & Matched OR & $\mathbf{9 5 \%} \mathbf{C l}$ & $\boldsymbol{P}$ \\
\hline IgM anti-EPCR & 16 & 3 & 14.0 & $1.8-106.4$ & .01 \\
IgG anti-EPCR & 13 & 4 & 4.3 & $1.2-15.2$ & .02 \\
FVL & 6 & 1 & 6.0 & $0.7-49.8$ & .10 \\
Prothrombin G20210A & 3 & 1 & 3.0 & $0.3-28.8$ & .34 \\
LACs & 7 & 1 & 7.0 & $0.9-56.9$ & .07 \\
IgM aCLs & 9 & 2 & 5.0 & $1.1-22.8$ & .04 \\
IgG aCLs & 1 & 0 & - & - & - \\
\hline
\end{tabular}

Anti-EPCR autoantibodies were categorized using the 95th percentile as a cutoff point for the variable in the control group. Levels of aCLs higher than $15 \mathrm{G}$ or $\mathrm{M}$ phospholipid units were considered positive.

—indicates not applicable.

IgM anti-EPCR, for IgG anti-EPCR (vice versa in case of $\mathrm{IgG}$ anti-EPCR); model 2, in which FVL was included instead of LACs. Using model 1, the OR of fetal death associated with $\operatorname{IgM}$ anti-EPCR levels above the 95th and 90th percentiles were 23.0 (95\% CI, 2.0-266.3) and 7.1 (95\% CI, 1.9-25.6), respectively. The ORs associated with IgG anti-EPCR levels exceeding the 95th and 90th percentiles were 6.8 (95\% CI, 1.2-38.4) and 3.5 (95\% CI, 1.9-11.8), respectively. In order to assess the risk associated with anti-EPCR autoantibodies whatever the isotype was, we created a composite variable that was either IgM or IgG level exceeding the 95th or 90th cutoff. In this way, we obtained adjusted the ORs of 10.9 (95\% CI, 2.2-54.7) and 6.7 (95\% CI, 2.1-21.2) with cutoffs at 95th and 90th percentiles, respectively. Using model 2 the results were similar (Table 2).

To determine whether the relationship between anti-EPCR autoantibody levels and the risk of fetal death was dose dependent, we stratified the subjects into quartiles according to the anti-EPCR levels, and OR for fetal death was calculated for each of the 3 upper quartiles using the lowest quartile as the reference. As Table 3 shows, the adjusted OR increased monotonically with the IgM anti-EPCR levels, suggesting a dose-response effect. However, the adjusted OR did not increase linearly with the IgG anti-EPCR levels, and thus a threshold response can be assumed.

Median (interquartile range) $\mathrm{IgM}$ and $\mathrm{IgG}$ anti-EPCR values were higher in patients with LACs than in patients without LACs (IgM, 54 [39-78] vs 82 [63-108] AUs, $P=.02$; IgG, 51 [37-64] vs 82 [56-117] AUs, $P=.01)$. Likewise, levels of IgM aCLs and anti-EPCR were positively correlated $(\operatorname{IgM}, \mathrm{r}=0.31, P<.0001$;

Table 2. Multivariate OR and their $95 \% \mathrm{Cl}$ for fetal death associated with anti-EPCR levels

\begin{tabular}{|c|c|c|c|c|c|c|}
\hline \multirow{2}{*}{$\begin{array}{c}\text { Anti-EPCR } \\
\text { antibodies and } \\
\text { percentile }\end{array}$} & \multicolumn{3}{|c|}{ Model 1} & \multicolumn{3}{|c|}{ Model 2} \\
\hline & $\begin{array}{c}\text { Matched } \\
\text { OR }\end{array}$ & $95 \% \mathrm{Cl}$ & $P$ & $\begin{array}{c}\text { Matched } \\
\text { OR }\end{array}$ & $95 \% \mathrm{Cl}$ & $P$ \\
\hline \multicolumn{7}{|l|}{$\lg M$} \\
\hline 95th & 23.0 & $2.0-266.3$ & .012 & 31.0 & $2.0-384.3$ & .007 \\
\hline 90th & 7.1 & $1.9-25.6$ & .003 & 9.1 & $2.4-34.7$ & .001 \\
\hline \multicolumn{7}{|l|}{$\lg G$} \\
\hline 95th & 6.8 & $1.2-38.4$ & .029 & 11.0 & $1.6-73.5$ & .013 \\
\hline 90th & 3.5 & $1.9-11.8$ & .040 & 5.5 & $1.5-20.2$ & .010 \\
\hline \multicolumn{7}{|l|}{ Either IgM or IgG } \\
\hline 95th & 10.9 & $2.2-54.7$ & .004 & 16.9 & $3-96$ & .001 \\
\hline 90th & 6.7 & $2.1-21.2$ & .001 & 10.4 & $2.9-37.4$ & $<.001$ \\
\hline
\end{tabular}

Anti-EPCR autoantibodies were categorized using the $95^{\text {th }}$ and $90^{\text {th }}$ percentiles as cutoff points for the variable in the control group. Model 1 was adjusted for G20210A prothrombin, LACs, aCLs, and, in case of $\operatorname{lgM}$ anti-EPCR, for IgG anti-EPCR (vice versa in case of IgG anti-EPCR). Model 2 is like model 1 except that FVL is included for adjustment instead of LACs. 
Table 3. OR for fetal death according to the anti-EPCR autoantibody levels

\begin{tabular}{lcccc}
\hline & $\begin{array}{c}\text { Cases/controls, } \\
\mathbf{n}\end{array}$ & $\begin{array}{c}\text { Matched } \\
\text { OR }\end{array}$ & $\begin{array}{c}95 \% \\
\mathbf{C l}\end{array}$ & $\begin{array}{c}\boldsymbol{P} \text { for } \\
\text { trend }\end{array}$ \\
\hline IgM & & & & \\
Q1, fewer than 33 AUs & $8 / 22$ & 1 & - & - \\
Q2, 33-52 AUs & $25 / 23$ & 3.1 & $1.0-9.6$ & - \\
Q3, 53-70 AUs & $19 / 21$ & 2.6 & $0.8-9.0$ & - \\
Q4, more than 70 AUs & $35 / 21$ & 4.4 & $1.4-14.3$ & .02 \\
Above p95, more than 99 AUs & $16 / 3$ & 45.2 & $2.9-662.4$ & - \\
IgG & & & & \\
Q1, fewer than 35 AUs & $18 / 22$ & 1 & - & - \\
Q2, 35-49 AUs & $18 / 23$ & 1.3 & $0.3-4.9$ & - \\
Q3, 50-57 AUs & $14 / 20$ & 0.8 & $0.2-2.7$ & - \\
Q4, more than 57 AUs & $37 / 22$ & 2.4 & $0.7-8.4$ & .12 \\
Above p95, more than 94 AUs & $13 / 4$ & 10.2 & $1.2-86.7$ & - \\
\hline
\end{tabular}

Patients and controls were stratified into quartiles according to the anti-EPCR level. OR for fetal death were calculated in the 2nd (Q2), 3rd (Q3), and 4th (Q4) quartiles, and 95th percentile (p95) compared with those in the 1st (Q1) quartile. Adjustment for variables associated with fetal death included in model 1 analysis was performed. - indicates not applicable.

IgG: $\mathrm{r}=0.24, P=.001)$. The crude OR of fetal death associated with LACs (7.0) or IgM aCLs (5.0) notably decreased and lost statistical significance when adjusting for anti-EPCR autoantibodies (3.1 [95\% CI, 0.3-27.9] and 2.3 [95\% CI, 0.4-12.8], respectively). This finding indicates that the classical APLs (LACs and aCLs) were not independent predictors of fetal death, the apparent increased risk associated with them being at least partly due to anti-EPCR autoantibodies.

No interaction could be seen among the analyzed variables (likelihood ratio test; $P>$.1). When we stratified these analyses by the number of pregnancies or by the period in which the fetal loss took place, the results did not substantially change.

\section{Discussion}

We developed an ELISA to detect anti-EPCR autoantibodies and we provide evidence supporting a strong association between these autoantibodies and the risk of fetal death. Thus for the first time the presence of anti-EPCR autoantibodies in human pathology has been demonstrated. First, we detected high levels of these antibodies in 5 patients within a group of APS patients showing thrombosis and LACs. Then, we provided evidence that anti-EPCR autoantibodies are sometimes able to hamper the PC activation, which should compromise anticoagulant and anti-inflammatory mechanisms. These PC activation-blocking antibodies may probably lead to a low level of APCs in vivo, a risk condition for thrombosis in itself, ${ }^{24}$ which would help to explain the multiple venous thrombosis episodes experienced by the patient who carried PC activationblocking antibodies in the APS group. Since we detected very high levels of IgM anti-EPCR in 2 APS women with previous history of multiple episodes of fetal death, we performed a matched casecontrol study to assess the risk of having a first episode of unexplained fetal death associated with the presence of anti-EPCR autoantibodies. This risk was dose-dependently higher as the level of IgM anti-EPCR increased. Very high levels of IgM anti-EPCR constituted a strong risk factor for the first episode of fetal death, with a 20-fold increase in relative risk. High levels of $\operatorname{IgG}$ anti-EPCR were also a strong risk factor, though weaker than IgM and without evidence for a dose-response trend. The broad confidence intervals obtained in the estimates of the relative risks were probably due to the reduced number of women exposed to levels higher than the 95th percentile. Nevertheless, when a lower cutoff (90th percentile) was set, the estimation of the risk was still high and the confidence intervals were narrowed, the same occurring when $\operatorname{IgM}$ and $\operatorname{IgG}$ isotypes were considered together. This finding strongly suggests that high levels of either IgM or IgG anti-EPCR are risk factors for fetal death. Furthermore, the associated risk might have been underestimated, since one of the criteria for including these patients in the study was to have neither a previous history of venous or arterial thrombosis nor previous obstetric complications, entities that theoretically could be associated with a higher prevalence of elevated anti-EPCR autoantibodies. It is not surprising that IgM anti-EPCR was more predictive than $\mathrm{IgG}$, since this is a common feature in obstetric complications, ${ }^{25-27}$ although a likely plausible biologic explanation for this phenomenon is not available. Known genetic risk factors, such as FVL or the prothrombin G20210A mutation, ${ }^{28}$ did not explain the increased risk of fetal death associated with anti-EPCR. Our findings are also not due to the effects of LACs or aCLs. It is noteworthy that while LACs and IgM aCLs were associated with an increased risk of fetal death in the univariate analysis, as previously reported, ${ }^{29,30}$ this association was markedly attenuated and lost its statistical significance in the multivariate model, where anti-EPCR autoantibodies were included in the analysis. AntiEPCR autoantibodies could thus be at least partly responsible for the pathologic effects observed in the patients showing LACs or aCLs. This notion is coherent with the fact that there is no plausible explanation about how LACs or aCLs might play a pathologic role by themselves, while anti-EPCR autoantibodies could indeed exert a variety of pathologic effects: the binding of autoantibodies to EPCR should be able to trigger the activation of the complement system. This will lead to a proinflammatory state with destruction of endothelium and trophoblast leading to a procoagulant state, and undesirable thrombosis and fetal loss as has recently been demonstrated in a mouse model of fetal death induced by APLs. ${ }^{31}$ On the other hand, since the correct function of the PC system is essential for promoting placental cell growth, ${ }^{10,15}$ it is conceivable that the anti-EPCR autoantibodies able to block the PC activation will exert an additional effect by reducing the APC concentration in such a manner that the placental development will be compromised. For these reasons, LACs and aCLs could be just innocent bystanders or mere markers of a more complex autoimmune disorder.

In conclusion, we have demonstrated for the first time the presence of $\operatorname{IgM}$ and $\operatorname{IgG}$ anti-EPCR in humans. High levels of these autoantibodies are associated with a higher risk of a first episode of fetal death. Anti-EPCR autoantibodies are often found in patients with APS as well. It is conceivable that these antibodies may exert a causative role in the thrombotic and the fetal death episodes occurring in these patients.

\section{References}

1. Levine JS, Branch DW, Rauch J. The antiphospholipid syndrome. N Engl J Med. 2002;346:752-763.

2. Arnout J. Antiphospholipid syndrome: diagnostic aspects of lupus anticoagulants. Thromb Haemost. $2001 ; 86: 83-91$.
3. Galli M, Comfurius P, Maassen C, et al. Anticardiolipin antibodies (ACA) directed not to cardiolipin but to a plasma protein cofactor. Lancet. 1990;335:1544-1547.

4. Rand JH, Wu XX, Andree HA, et al. Pregnancy loss in the antiphospholipid-antibody syndrome-a possible thrombogenic mechanism. N Engl J Med. 1997;337:154-160.

5. Esmon CT. Regulation of blood coagulation. Biochim Biophys Acta. 2000;1477:349-360. 
6. Oosting JD, Derksen RH, Bobbink IW, Hackeng TM, Bouma BN, de Groot PG. Antiphospholipid antibodies directed against a combination of phospholipids with prothrombin, protein $\mathrm{C}$, or protein S: an explanation for their pathonegic mechanism? Blood. 1993;81:2618-2625.

7. Fukudome K, Esmon CT. Identification, cloning, and regulation of a novel endothelial cell protein $\mathrm{C} /$ activated protein $\mathrm{C}$ receptor. J Biol Chem. 1994;269:26486-26491.

8. Oganesyan V, Oganesyan N, Terzyan S, et al The crystal structure of the endothelial protein $\mathrm{C}$ receptor and a bound phospholipid. J Biol Chem. 2002;277:24851-24854.

9. Laszik Z, Mitro A, Taylor FB Jr, Ferrell G, Esmon CT. Human protein $C$ receptor is present primarily on endothelium of large blood vessels: implications for the control of the protein $C$ pathway. Circulation. 1997;96:3633-3640.

10. Isermann $B$, Sood R, Pawlinski R, et al. The thrombomodulin-protein $\mathrm{C}$ system is essential for the maintenance of pregnancy. Nat Med. 2003;9: 331-337.

11. Stearns-Kurosawa DJ, Kurosawa S, Mollica JS, Ferrell GL, Esmon CT. The endothelial cell protein $\mathrm{C}$ receptor augments protein $\mathrm{C}$ activation by the thrombin-thrombomodulin complex. Proc Nat Acad Sci U S A. 1996;93:10212-10216.

12. Riewald M, Petrovan RJ, Donner A, Mueller BM, Ruf W. Activation of endothelial cell protease activated receptor 1 by the protein $\mathrm{C}$ pathway. Science. 2002;296:1880-1882.

13. Cheng T, Liu D, Griffin JH, et al. Activated protein C blocks p53-mediated apoptosis in ischemic human brain endothelium and is neuroprotective. Nat Med. 2003;9:338-342.

14. Ruf W. PAR1 signaling: more good than harm? Nat Med. 2003;9:258-260

15. Gu JM, Crawley JT, Ferrell G, et al. Disruption of the endothelial cell protein $C$ receptor gene in mice causes placental thrombosis and early em- bryonic lethality. J Biol Chem. 2002;277:4333543343.

16. Wilson WA, Gharavi AE, Koike T, et al. International consensus statement on preliminary classification criteria for definite antiphospholipid syndrome: report of an international workshop. Arthritis Rheum. 1999;42:1309-1311.

17. Brandt JT, Barna LK, Triplett DA. Laboratory identification of lupus anticoagulants: results of the Second International Workshop for Identification of Lupus Anticoagulants: on behalf of the Subcommittee on Lupus Anticoagulants/Antiphospholipid Antibodies of the ISTH. Thromb Haemost. 1995;74:1597-1603.

18. Gris JC, Quere I, Monpeyroux F, et al. Case-control study of the frequency of thrombophilic disorders in couples with late foetal loss and no thrombotic antecedent-the Nîmes Obstetricians and Haematologists Study 5 (NOHA5). Thromb Haemost. 1999;81:891-899.

19. Simmonds RE, Lane DA. Structural and functional implications of the intron/exon organization of the human endothelial cell protein C/activated protein $\mathrm{C}$ receptor (EPCR) gene: comparison with the structure of CD1/major histocompatibility complex alpha1 and alpha2 domains. Blood. 1999;94:632-641.

20. Maruyama S, Kubagawa H, Cooper MD. Activation of human $B$ cells and inhibition of their terminal differentiation by monoclonal anti-mu antibodies. J Immunol. 1985;135:192-199.

21. Ye X, Fukudome K, Tsuneyoshi N, et al. The endothelial cell protein $\mathrm{C}$ receptor (EPCR) functions as a primary receptor for protein $\mathrm{C}$ activation on endothelial cells in arteries, veins, and capillaries. Biochem Biophys Res Commun. 1999;259:671677.

22. Fukudome K, Kurosawa S, Stearns-Kurosawa DJ, He X, Rezaie AR, Esmon CT. The endothelial cell protein $C$ receptor: cell surface expression and direct ligand binding by the soluble receptor. J Biol Chem. 1996;271:17491-17498.
23. Regan LM, Stearns-Kurosawa DJ, Kurosawa S, Mollica J, Fukudome K, Esmon CT. The endothelial cell protein $C$ receptor: inhibition of activated protein $\mathrm{C}$ anticoagulant function without modulation of reaction with proteinase inhibitors. J Biol Chem. 1996;271:17499-17503.

24. España F, Vayá A, Mira Y, et al. Low level of circulating activated protein $\mathrm{C}$ is a risk factor for venous thromboembolism. Thromb Haemost. 2001; 86:1368-1373.

25. Gris JC, Quere I, Sanmarco M, et al. Antiphospholipid and antiprotein syndromes in non-thrombotic, non-autoimmune women with unexplained recurrent primary early foetal loss: the Nîmes Obstetricians and Haematologists Study- $\mathrm{NOHA}$. Thromb Haemost. 2000;84:228-236.

26. Gris JC, Perneger TV, Quere I, et al. Antiphospholipid/antiprotein antibodies, hemostasis-related autoantibodies, and plasma homocysteine as risk factors for a first early pregnancy loss: a matched case-control study. Blood. 2003;102: 3504-3513.

27. Gris JC, Amadio C, Mercier E, et al. Anti-protein Z antibodies in women with pathologic pregnancies. Blood. 2003;101:4850-4852.

28. Martinelli I, Taioli E, Cetin I, et al. Mutations in coagulation factors in women with unexplained late fetal loss. N Engl J Med. 2000;343:1015-1018.

29. Lockshin MD, Druzin ML, Goei S, et al. Antibody to cardiolipin as a predictor of fetal distress or death in pregnant patients with systemic lupus erythematosus. N Engl J Med. 1985;313:152156.

30. Oshiro BT, Silver RM, Scott JR, Yu H, Branch DW. Antiphospholipid antibodies and fetal death. Obstet Gynecol. 1996;87:489-493.

31. Girardi G, Berman J, Redecha P, et al. Complement $\mathrm{C} 5$ a receptors and neutrophils mediate fetal injury in the antiphospholipid syndrome. J Clin Invest. 2003:112:1644-1654. 